\title{
Coalition Building and the Intervention Wheel to Address Breast Cancer Screening in Hmong Women
}

\author{
Jill L. Depke, APNP, AOCNP and Adedayo A. Onitilo, MD, MSCR, FACP
}

\section{Keywords:}

Breast cancer screening

Coalition building

Hmong women

Intervention wheel

Corresponding Author:

Jill L. Depke, APNP, AOCNP

Marshfield Clinic - Weston Center

Cancer Care-Hematology/Oncology

3501 Cranberry Boulevard

Weston, WI 54476

Tel: $715-393-1400$

Fax: 715-393-1399

E-mail: depke.jill@marshfieldclinic.org

Received: September 7, 2010

Revised: January 18,2011

Accepted: January 21, 20II

doi: $10.3121 / \mathrm{cmr} .2011 .964$
B

reast cancer is the most frequently diagnosed cancer among women in the United States, where 1 in 8 women will face breast cancer in their lifetime. ${ }^{1}$ In 2010, this translates to approximately 207,090 women who will be diagnosed with this disease, and about 54,010 who will die of breast cancer. ${ }^{1}$

While Caucasian women have the highest incidence of breast cancer in comparison to any other cultural group, Asian American and Pacific Islander (AAPI) women, in aggregate, are more likely to die from breast cancer than any other ethnic group. ${ }^{2,3}$ Furthermore, Asian American females are the first American population to experience cancer as the leading cause of death in the United States. ${ }^{3,4}$ Death rates have increased in AAPI women by $200 \%$ since $1990 .{ }^{5}$ In addition, AAPI women collectively have the lowest screening rates of all ethnic populations, with roughly $45 \%$ reporting that they have ever had a mammogram. ${ }^{4}$ Hmong women, an ethnic group within the AAPI population, are among those at the highest risk for health problems due to high rates of poverty, language isolation, and cultural barriers. ${ }^{3}$

A national health initiative, Healthy People 2020,6 , was created to increase health promotion and disease prevention that address issues such as these. A primary objective identifies cancer as one of the ten leading health indicators. The goal is to reduce the number of new cases, as well as the illness, disability, and death caused by cancer. The ability to decrease cancer death rates depends on the existence and application of various types of resources, and providing culturally and linguistically appropriate information on prevention, early detection, and treatment to the public is essential in order to meet this goal. ${ }^{6}$

A new and more specific goal presented by Healthy People 2020 is to decrease the incidence of late stage breast cancer. This is of particular importance in Asianborn Asian American women with breast cancer, in which approximately $79 \%$ have a greater proportion of tumors larger than $1 \mathrm{~cm}$ at diagnosis. ${ }^{7}$ It has been shown that many tumors greater than $1 \mathrm{~cm}$ have lymph node involvement and are considered later stage disease.

Another goal established from Healthy People 2020 is to increase the proportion of women aged 40 or older who have received a breast cancer screening based on the most recent guidelines. It is essential that a high percentage of females over age 40 comply with these mammographic screening recommendations in order to reduce breast cancer burden and death rates. 
Given the diversity and health disparities of the Hmong population, it is important to develop culturally competent health promotion programs, including breast cancer screening, to address and support the health needs of this group. Coalitions can be the cornerstones of creating successful change within a community. They represent a range of local interests and bring together organizations and individuals to build a foundation that works to influence social change of a communal concern. It is conceivable that coalition building can establish an effective breast cancer-screening program within the Hmong population.

\section{Background}

Asian American and Pacific Islanders comprise over 50 distinct ethnicities, speak over 100 languages and dialects, and encompass the fastest growing minority population in the United States. ${ }^{2}$ The cultural groups that make up the AAPI communities are often grouped together and considered as a single homogeneous entity. In reality, these communities are diverse in language, culture, and history, and such differences can influence access to health information, health maintenance opportunities, health care, and health outcomes.

Seventy percent of the United States Asians are those who entered the United States in three distinct waves: before 1975, between 1975-1979, and 1980 and later. ${ }^{8}$ According to the US Census Bureau, the population of AAPI has increased by $72 \%$ since 1990 , from approximately 1.5 million individuals in 1990 to more than 11.2 million in $2000 .^{8}$

One of these groups, the Hmong, has sought refuge from persecution. The Hmong fled Laos after the Vietnam War when the communists targeted them for genocide due to their alliance with the United States Central Intelligence Agency (CIA) for harboring and helping coalition forces from planes that went down during the war. ${ }^{9-11}$ Geographically, Hmong immigrants have tended to settle in primarily three refugeefriendly states: California, Minnesota, and Wisconsin. ${ }^{8}$

The Hmong are a group of people who lived in mountainous areas of Laos where their isolation prohibited their contact to other cultures. ${ }^{9-11}$ They are a traditional people with no real written language prior to coming to the U.S., and since their language had been primarily verbal, the transition to a culture based on written words made acculturation even more difficult. $^{12}$

\section{Hmong Beliefs and Health Practices}

The Hmong culture encompasses an array of health beliefs and practices. It combines Chinese medicine, Protestant Christian beliefs, spirit illness and soul loss beliefs. The Hmong believe to speak of death, illness, or disability will cause it to occur. It is also their belief that when Hmong die, they must have all their body parts, otherwise they will be "deformed" when they are reincarnated in the next generation. ${ }^{13}$
In Laos, many of the Hmong used shamans and a belief in animal spirits in their folk healing. Shamans, who are thought to communicate directly with the spirits, are important healers and leaders in the Hmong community. The shaman performs an initial ceremony by going into the spirit world to find out where the soul is and what payment or sacrifice the soul requires to return to the individual. ${ }^{10}$

Within the Hmong culture in Laos, age 30 was considered old because death often occurred at a young age. ${ }^{13}$ In the United States, Hmong aged 30 or older are considered elderly and worthy of strong respect. ${ }^{13}$ The Hmong culture extends far beyond the immediate family and does not view individuals separately from their family or clan. All decision making, including health care decisions of the Hmong, is hierarchical. ${ }^{10,13}$ Their way of life emphasizes group welfare over an individual's needs, accounting for why many Hmong families may feel pressured by providers into making health care decisions, including treatment, because family may need to wait for other members to travel long distances to make these decisions. ${ }^{10}$

The Hmong often fear Western medical treatments and mistrust health care providers. ${ }^{10}$ They also desire to present themselves in a positive light, and they consider it impolite to tell a health care provider that they are not accustomed to taking medications as prescribed. ${ }^{10}$ They will often respond "yes" in a respectful way to indicate they heard what was said, but not to imply that they understood what was said, agree with, or would follow any recommendations that were given. ${ }^{10,13}$

\section{Hmong Belief on Breast Cancer Screening}

Cancer is the second leading cause of death among every racial/ethnic group in the United States except for AAPI, for whom it is first. ${ }^{4}$ The practice of merging AAPI health data into a single category hides significant rate variations, and the groups at highest risk for late cancer detection and treatment become invisible. ${ }^{5}$ Few studies have been published on Hmong breast screening practices. A study of Hmong women in southern California illustrates the fact that they have one of the lowest screening rates of all ethnic populations. ${ }^{5}$ A preintervention survey showed that only $45.8 \%$ of all Hmong women had heard of a mammogram, and only $28.6 \%$ of the women had had a mammogram..$^{5}$ Another study found that only $51 \%$ of Hmong women surveyed had ever performed a self-breast exam. ${ }^{3}$ Furthermore, in screening practices of Asian American respondents aged 40 or older, only $52 \%$ had ever had a clinical breast exam, and only $30 \%$ had ever had mammography. ${ }^{3}$

Many Asian Americans underutilize cancer-screening tests. Among the barriers to breast cancer screening indicated by Asian American females in earlier studies were modesty, reluctance to disrobe for a breast exam (especially performed by male physicians), and language barriers. ${ }^{14}$ The Hmong value their privacy, making physical examinations by a 


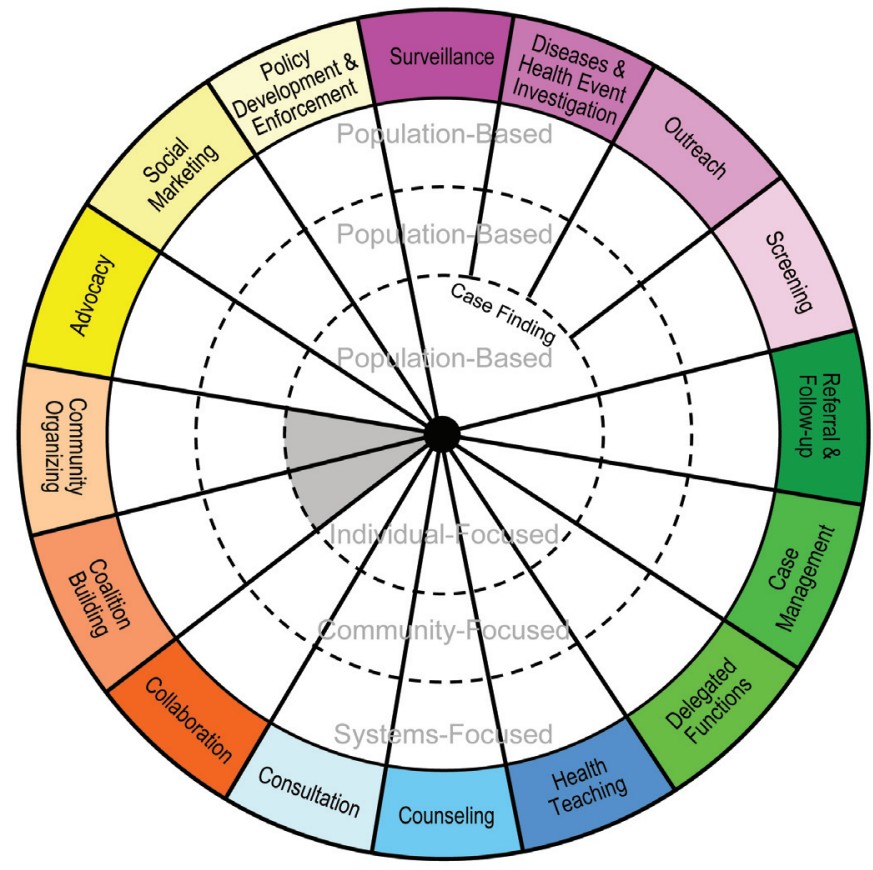

Figure 1. Public Health Interventions: Intervention Wheel. ${ }^{19}$ Interventions are actions that public health nurses take on behalf of individuals, families, systems, and communities to improve or protect health status. Reprinted with permission from the Minnesota Department of Health, Section of Public Health Nursing. 2001. Public Health Nursing Interventions: Applications for Public Health Nursing Practice. (http://www. health.state.mn.us/divs/cfh/ophp/resources/docs/wheel.pdf).

physician, especially one of the opposite gender, an issue. Even with same-gender clinicians, Hmong women may not permit examinations. ${ }^{13}$

Nearly one-third of AAPIs are isolated linguistically, with significant variations among subgroups such as the Hmong with 55.8\% language isolation. Moreover, non-English speaking AAPIs are typically excluded from national surveys, such as the Behavioral Risk Factor Surveillance System, since these are often administered in English or Spanish only, and they tend to have the lowest screening rates. ${ }^{5}$

Demographic characteristics that affect health care access include education, income, age, language, and cultural differences, and all had a significantly greater negative impact on screening rates among AAPI than on white women. ${ }^{5}$ Major variations among AAPI subgroups in demographic risk factors for low rates of cancer screening, including low education, income levels, and age, were identified. ${ }^{5}$ The number of women with less than four years of education was $2 \%$ nationally and $6 \%$ for AAPI women. ${ }^{5}$ The poverty rate for whites was $6.6 \%$, whereas the rate for all Southeast Asian groups was more than $66 \%,{ }^{5}$ and the median age in the United States is 33.4 years, yet the median age for the Hmong is only 12.5 years. $^{5}$
Many Asian Americans have emigrated from countries with the overall lowest breast cancer rate in the world. ${ }^{15}$ Studies suggest that exposure to the American lifestyle increases breast cancer risk among immigrants from Asia. ${ }^{16}$ The longer that foreign-born AAPI women reside in the United States, the more likely that their risk of developing breast cancer will come close to that of American-born women. ${ }^{17}$ As lifestyles of the Hmong change due to Western influences, their cancer incidence rates, especially among descendents, may change to more closely reflect the cancer incidence rates of the native United States population. For example, breast cancer incidence rates in the U.S.-born Japanese population now approach the rates in the non-Hispanic white population. ${ }^{18}$

The breast health needs of Hmong women, through screening, can be addressed with the use of coalition building and the Intervention Wheel (figure 1). ${ }^{19}$ The Intervention Wheel is a distinctive way of assessing a population, like the Hmong, who have an actual health need of breast cancer screening. Coalition building, one of the public health interventions in the Intervention Wheel, can be effectively utilized to gain entrance and better insight into this unique population. The importance of breast cancer screening through health promotion, screening efforts and coalition building, are examined with this population.

\section{The Intervention Wheel}

The Intervention Wheel is a population-based practice model that includes three levels of practice (community, systems, and individual/family) and 17 public health interventions. Each intervention and practice level contributes to improving the health of the population. ${ }^{20}$ The Intervention Wheel was initially introduced in 1998 by the Minnesota Department of Health, Section of Public Health Nursing. ${ }^{19}$ The model was subjected to a thorough critique process through a systematic review that established the validity of this model. ${ }^{20}$

Population-based public health practice focuses on entire populations that actually or potentially hold similar health concerns or characteristics. While public health programs have conventionally been problem focused, there is rising recognition that promoting protective factors is just as important as reducing risk factors. ${ }^{20}$ Health promotion targets essentially "well" populations. Promoting the health practices of breast cancer screening includes health education, "breast self-awareness", clinical breast exam, and mammography. ${ }^{21}$ "Breast self-awareness" is becoming familiar with the normal appearance and feel of the breasts and reporting changes promptly, in lieu of self breast examination. ${ }^{21}$

Community assessment will assist in identification of the health needs of a population, and this assessment will identify cultural and ethnic characteristics that must be considered in order to develop culturally relevant interventions. ${ }^{20}$ Utilizing coalition building through use of the Intervention Wheel will assist in identifying barriers, but unfortunately, multiple obstacles exist for breast cancer screening in Hmong women. 
Table 1. Eight steps to coalition building in the Intervention Wheel. ${ }^{19}$

- Decide whether or not to come together

- $\quad$ Recruiting the right members

- Devise a set of preliminary objectives and activities

- $\quad$ Convene the coalition to present a strong proposal for the coalition's structure, mission, membership, and response at the initial meeting

- Anticipate human, material, and financial resources required to accomplish the goal

- Select an appropriate structure including life expectancy of the coalition, location, frequency, and length of meetings, membership criteria, decision-making process, agenda setting, and rules for participation

- Maintain the vitality of the coalition

- Make improvements through evaluation

The principle issue is the language barrier which can greatly affect Hmong women by adding to the cultural separation; however, the use of interpreters can assist in breaking down this barrier.

Additionally, the lack of a basic understanding about human anatomy can be another setback. The Hmong language does not have terms that translate medical physiology and anatomy, nor do they have a word for "cancer." The Hmong believe discussing a problem that does not exist may place a hex on the patient and actually cause the disease to occur. Because of this, Hmong women may possess fears about cancer in their bodies or hold a fatalistic view of cancer. Therefore, extra measures need to be taken by health care providers to avoid mistrust and fear of Western medicine. ${ }^{10}$ Education using pictures or visual aids of the human body can help to overcome this issue.

Hmong women may be unable to keep screening appointments because they are the primary caretakers for children or grandchildren. The traditional Hmong woman's role is to be the "hands and feet" of her husband, spending time assisting her husband and the extended family, doing the household chores, and raising the children. ${ }^{22}$ Extended families often live together in the same household, and women do not have time to take care of their own health care needs because of their responsibility to their family. They may also lack transportation to get to an appointment.

Finally, Hmong women may have financial concerns. Federaland state-funded programs, such as the Well Women's Project, can help low-income Hmong women pay for mammograms, as well as local health departments that offer free mammograms to ethnic populations on specific days of the month. The coordination of a mobile health screening van that goes into the community where Hmong women live may provide free or reduced cost mammograms to women who meet screening criteria. Timely follow-through regarding abnormal results is also an important aspect of breast cancer screening and extremely important in this population.

The Intervention Wheel can assist in the development of structured programs and practices to meet health needs through breast cancer screening in this population. Public health interventions can be directed at an entire population within a community, the systems that affect the health of those populations, or the individuals and families within those populations. ${ }^{20}$ A population system-focused practice changes organizations, policies, laws, and power configurations. System changes can have a more effective and longer lasting effect on a population. Facilitation of coalitions is an important strategy in a system-focused practice. Populationbased community-focused practice changes community norms, attitudes, awareness, practices, and behaviors. ${ }^{20}$ Coalition building is important in order to change a community's view on breast cancer screening. A population individual-focused practice changes the knowledge, attitudes, beliefs, practices, and behaviors of individuals. Teaching women breast cancer screening through health education, "breast self-awareness," clinical breast exam, and use of mammography is an example of this practice.

\section{Health Promotion Through Coalition Building: \\ A Breast Cancer Screening Program}

Coalition building is initiated through promoting and developing alliances among organizations or constituencies for a common purpose and builds linkages, solves problems, and/or enhances local leadership to address health matters. ${ }^{20}$ The decision to form a coalition is based on recognition of a community's need to respond to a perceived health issue and whether a coalition would help fulfill the goals of an organization $^{19}$ (table 1).

The goal of the coalition should determine the type of members. Given the goal of increasing breast cancer screening in Hmong women through education and reducing their barriers to screening, members should include leaders within the Hmong population, female Hmong interpreters, members of the local Hmong Association, and business leaders where Hmong women are employed, to help gain a better insight into the Hmong culture. Representatives from the American Cancer Society and the Department of Health and Human Services should also be included. Female physicians, advanced practice nurses, and nurses within the community representing primary care, women's health, and oncology care, would be additionally important members. It would also be essential to 
include Hmong health care practitioners. If the coalition's agenda is organized without community input, an important member is missing. Male leaders, given their importance in making decisions in the family/clan, need to be included, because their influence on members could facilitate Hmong women's awareness and interest in seeking breast cancer screening. It is important for practitioners to work within the patriarchal culture with efforts to empower the Hmong to advocate for optimal healthcare for themselves and their families. ${ }^{23}$

An example of utilizing leaders in the Hmong community for cancer health promotion was established in California. A course called "Cancer Awareness 101" is the centerpiece of the Asian American Network for Cancer Awareness, Research and Training (AANCART) Hmong outreach. ${ }^{24}$ It is designed to give members of the Hmong community, particularly the elders and shaman who often serve as medical decisionmakers for their families, the same baseline cancer knowledge that more mainstream Americans grow up knowing. ${ }^{24}$

Another example of a breast cancer screening awareness study among the Hmong community was the "Life Is Precious" study that took place in Fresno and San Diego, California. It developed culturally and linguistically appropriate flipcharts, brochures, and videos, and also educated men to support their wives' breast cancer screenings. ${ }^{25}$

Identifying a primary goal and its objectives is a crucial step in coalition building. The primary goal of this health promotion project would be to increase breast cancer screening in Hmong women through the use of health education, "breast self-awareness," clinical breast exam, and mammography. All members should have a stake in the outcomes, avoid turf struggles, make objectives compelling through short-term reachable goals, keep assignments simple and achievable, and set limits. Encouraging each member to respond to a prepared proposal is usually more productive than expecting the group to create the proposal. ${ }^{19}$

The coalition must anticipate the human, material, and financial resources required to accomplish their goal. Effective coalitions generally require minimal financial spending for materials and supplies, but substantial time and energy commitments of members. In calculating resources, it is important to estimate categories such as clerical, meeting support, membership support, research and fact gathering, public relations and information, coordination of activities, and fund raising or grant proposals. ${ }^{19}$ Hmong translators will play an important role in developing breast cancer screening information including pamphlets for distribution to Hmong women.

Selecting an appropriate structure of the coalition including life expectancy, location, frequency and length of meetings, membership criteria, decision-making process, agenda- setting, and rules for participation is vital to its productivity. ${ }^{19}$ Keeping the purpose of the coalition in mind will assist members in keeping on track with the objectives set by the group.

Maintaining the vigor of the coalition should be an integral component in a coalition. Involving members who are naturally positive and energetic can bring vitality to the group. Incorporating members of the Hmong community to describe important aspects of the culture would assist others in the coalition to better understand the cultural differences, which will ultimately affect the success of Hmong participation in the health promotion program.

Evaluation should be an ongoing process in coalition building and should involve both process (formative) and outcome (summative) evaluation. Use of surveys, analysis of the program content, and whether the goals of the coalition were achieved are important in the evaluation of the objectives. ${ }^{19}$ Utilizing interviews of a small sample of Hmong women of various ages would provide a position for establishing the health promotion program of breast cancer screening. A recent study on Hmong women's perceptions and beliefs on screening mammography highlighted that breast health messages from lay and professional community have significant influence on Hmong women's decisions to seek preventative health care. Furthermore, breast health education offers benefits of an increased awareness of disease formation and the need for screening; however, it does not necessarily lead to a decision to seek preventative breast health care. ${ }^{11}$

\section{Conclusion}

Utilizing the Intervention Wheel is a unique approach to establish coalition building as a method of addressing the breast health needs of Hmong women. Building a strong coalition to increase breast cancer screening in Hmong women through education and a reduction of their barriers to screening is necessary. Hmong women are among those at the highest risk for health problems due to high rates of poverty, language isolation, and cultural barriers. ${ }^{3}$ Asian American females are the first American population to experience cancer as the leading cause of death in the United States. ${ }^{3,4}$ Not diagnosing breast cancer until late stages leads to increased morbidity in Hmong women. Developing a culturally sensitive breast cancer screening program is essential to meeting the goal of increasing breast care through health education, "breast self-awareness," clinical breast exam and mammography in the Hmong population.

\section{Acknowledgements}

The authors thank the Marshfield Clinic Research Foundation for providing assistance in the preparation of this manuscript through the services of the Office of Scientific Writing and Publications. 


\section{References}

1. American Cancer Society. Cancer facts \& figures 2010. Available at: http://www.cancer.org. Accessed November 21, 2010.

2. Fu L, Macabeo A, Matsunaga D, Nguyen T-U, Rezai K, KagawaSinger M. Providing culturally tailored breast and cervical cancer programs for Asian and Pacific Islander women: a case study of a Filipino community in Honolulu. Californian J Health Promot 2003;1:40-53.

3. Tanjasiri S, Kagawa-Singer M, Foo M, Chao M, Linayao-Putman I, Lor Y, Xiong Y, Moua M, Nguyen J, Vang X. Breast cancer screening among Hmong women in California. J Cancer Educ 2001;16:50-54.

4. Asian Americans and cancer. Intercultural Cancer Council. Available at: http://iccnetwork.org/cancerfacts/cfs3.htm. Accessed November 10, 2004.

5. Kagawa-Singer M, Pourat N. Asian American and Pacific Islander breast and cervical carcinoma screening rates and healthy people 2000 objectives. Cancer 2000;89:696-705.

6. Healthy People 2020; Cancer. Centers for Disease Control and Prevention, National Institutes of Health. Available at: http://www.healthypeople.gov. Accessed November 21, 2010.

7. Hedeen A, White E, Taylor V. Ethnicity and birthplace in relation to tumor size and stage in Asian American women with breast cancer. Am J Public Health 2009;89:1248-1252.

8. United States Bureau of the Census. U.S. Census Report (2000). Washington, DC: Government Printing Office; 2000.

9. Hamilton-Merritt J. Tragic mountains: The Hmong, the Americans, and the secret war for Laos, 1942-1992. $1^{\text {st }}$ ed. Bloomington, IN: University of Indiana Press; 1993.

10. Johnson SK. Hmong health beliefs and experiences in the western health care system. J Transcult Nurs 2002; 13:126-132.

11. Yang D. The Hmong: enduring traditions. In: Lewis J, ed. Minority cultures of Laos: Kammu, Lua', Lahu, Hmong, and Iu-Mein. Folsum, CA: Folsum Cardova Unified School District;1992. 249-327.

12. Yee BWK. Health and health care of southeast Asian American elders: Vietnamese, Cambodian, Hmong, and Laotian Elders. Available at: http://www.stanford.edu/group/ethnoger/ southeastasian.html. Accessed November 10, 2004.

13. Parker M, Kiatoukaysy L. Culturally responsive health care: the example of the Hmong in America. J Am Acad Nurse Pract 1999;11:511-518.

14. Jenkins C, McPhee S, Bird J, Pham G, Nguyen B, Nguyen T, Lai KQ, Wong C, Davis TB. Effect of a media-led education campaign on breast and cervical cancer screening among Vietnamese-American women. Prev Med 1999;28:395-406.

15. Jemal A, Tiwari R, Murray T, Ghafoor A, Samuels A, Ward E, Feuer EJ, Thun MJ; American Cancer Society. Cancer statistics, 2004. CA Cancer J Clin 2004;54:8-29.

16. Ziegler R, Hoover R, Pike M, Hildesheim A, Nomura A, West DW, Wu-Williams AH, Kolonel LN, Horn-Ross PL, Rosenthal JF, Hyer MB. Migration patterns and breast cancer risk in Asian American women. J Natl Cancer Inst 1993;85:1819-1827.

17. Polek C, Klemm P, Hardie T, Wheeler E, Birney M, Lynch K. Asian/Pacific Islander American women: age and death rates during hospitalization for breast cancer. Oncol Nurs Forum 2004;31:E69-74.

18. Ross J, Xie Y, Kiffmeyer W, Bushhouse S, Robison L. Cancer in the Minnesota Hmong population. Cancer 2003; 97:3076-3079.

19. Minnesota Department of Health, Section of Public Health Nursing. Public health nursing interventions: Applications for public health nursing practice, March 2001. Available at: http://www.health.state.mn.us/divs/cfh/ophp/resources/docs/ phinterventions_manual2001.pdf. Accessed May 11, 2005.
20. Keller L, Strohschein S, Lia-Hoagberg B, Schaffer M. Population-based public health interventions: practice-based and evidence-supported. Part I. Public Health Nurs 2004;21:453-468.

21. Griffin JL, Pearlman MD. Breast Cancer Screening in Women at Average Risk and High Risk. Obstet Gynecol 2010;116:1410-1421.

22. Vang PC. Using mammography screening: Hmong women's perspectives and beliefs. Hmong Studies Journal. Available at: http://hmongstudies.org/VangHSJ10.pdf. Accessed December 10, 2009.

23. Baisch MJ, Vang PC, Peterman BR. An exploration of Hmong women's perspectives on screening for and treating cancer in their community. Asian Nurs Res 2008;2:82-91.

24. Cancer awareness 101. Synthesis, a publication of the UC Davis Cancer Center. Vol. 7, No. 2 Fall/Winter. University of California-Davis. Available at: http://www.ucdmc.ucdavis. edu/synthesis/Archives/fall_winter_05/features/outreach. html. Accessed May 10, 2005.

25. Tanjasiri SP, Kagawa-Singer M, Foo MA, Chao M, LinayaoPutman I, Nguyen J, Pirumyan G, Valdez A. Designing culturally and linguistically appropriate health interventions: the "Life Is Precious" Hmong breast cancer study. Health Educ Behav 2007;34:140-153.

\section{Author Affiliations}

Jill L. Depke, APNP, AOCNP and

Adedayo A. Onitilo, MD, MSCR, FACP

Marshfield Clinic-Weston Center

Cancer Care-Hematology/Oncology

3501 Cranberry Blvd.

Weston, WI 54476 USA 